\title{
Seroprevalence and molecular detection of Newcastle disease virus in backyard chickens in Tripoli, Libya
}

\author{
Emhemed A. Gedara ${ }^{1 \S}$, Hesham A. Alseed ${ }^{2 \S}$, Abdulwahab M. Kammon $^{3}$, Abdunaser S. Dayhum $^{3}$, \\ Mohamed A. Benothman ${ }^{3}$, Soleman O. Al-Garib ${ }^{3 \dagger}$ and Ibrahim M. Eldaghayes ${ }^{3 *}$ \\ ${ }^{1}$ Department of Microbiology, Faculty of Veterinary Sciences, University of Aljfara, Aljfara, Libya \\ ${ }^{2}$ Department of Microbiology, Higher Institute of Medical Technology, Tripoli, Libya \\ ${ }^{3}$ Faculty of Veterinary Medicine, University of Tripoli, Tripoli, Libya \\ ${ }^{\S}$ Contributed equally in this study \\ tDeceased
}

\begin{abstract}
Background: Newcastle disease (ND) is a viral disease that affecting many avian species all over the world.

Aim: ND has been successfully controlled by the vaccination of commercial poultry in Libya. However, there was a lack of information about the situation of ND in backyard chickens. Therefore, this study determined the prevalence of ND in backyard chickens in different locations of Tripoli.

Methods: A total number of 280 cloacal swabs (190 in summer and 90 in winter) and 412 sera were collected from non-vaccinated backyard chicken flocks in different geographical locations within the area of Tripoli namely Qasr Ben Ghashier, Al-Sawani, Souq Al-Gomaa, Tajourah, Ein Zara, and Janzour. Cloacal swabs and sera were tested by real time polymerase chain reaction (PCR) and ELISA, respectively.

Results: The prevalence of ND virus (NDV) infection in backyard chickens in different locations of Tripoli during summer and winter was $45 \%$ using real-time reverse transcription-PCR. Except in Qasr Ben Ghashier, the prevalence in summer season was significantly higher than in winter $\left(X^{2}=46.13, p \geq 0.00001\right)$. ELISA test revealed 218 positive out of 412 tested samples with total prevalence of 53\% across the city of Tripoli in all regions. Obviously, Qasr Ben Ghashier had significantly $\left(X^{2}=74.09, p \geq 0.00001\right)$ the highest prevalence $(82 \%)$ of NDV specific antibodies followed by Tajourah $(68 \%)$.

Conclusion: This study demonstrated the situation of ND in backyard chicken highlighting the necessity of a comprehensive vaccination plan for backyard chickens.

Keywords: Backyard chickens, ELISA, Newcastle disease, Prevalence, Real time PCR.

\section{Introduction}

The history of Newcastle disease (ND) began in 1926 with the depiction of fatal disease syndrome through diversifying sites at different geographical locations worldwide, Newcastle upon Tyne, England and the island of Java, Indonesia (Kraneveld, 1926; Doyle, 1927). ND virus (NDV), or avian paramyxovirus type 1 (APMV-1), belongs to the genus Avulavirus, subfamily Paramyxovirinae, and family Paramyxoviridae (Alexander, 1997). The genome of NDV is negative sense, single-stranded RNA (Alexander and Senne, 2008). ND has a global concern due to its significant characterizations, such as respiratory, nervous, gastrointestinal, and reproductive impairment leading to severe economic losses and threatening among intensive poultry-farming systems and free-range establishments (Tiwari et al., 2004). In addition, the disease occurs in three pathotypes, namely, lenthogenic, mesogenic, and velogenic, reflecting increasing levels of virulence. The velogenic strains (most virulent) are further subdivided into neurotropic

and viscerotropic NDVs (Alexander, 1997). As there are variations in the virulence of NDV strains, the rate of mortality and morbidity in chickens also varies with decrease in egg production in laying hens (Alexander, 1997; Alexander and Senne, 2008).

Severe outbreaks of ND has occurred in Libyan poultry industry during March 2013, and the causative agent of APMV-1 was isolated and characterized (Kammon et al., 2015). The virus was inoculated in the allantoic fluid of embryonated eggs and confirmed by real-time reverse transcription-PCR (rRT-PCR). The genetic sequencing of the APMV-1/chicken/ Libya/13VIR/7225-1/2013 isolate revealed the presence of a velogenic APMV-1 that belongs to the genotype VIIi in class II (Kammon et al., 2015). In the meanwhile, a new control strategy was adopted by the Veterinary Authority depended on the use of live and inactivated ND vaccines. This strategy has successfully reduced the threat of ND in commercial poultry but more outbreaks emerged in unvaccinated backyard birds. In March 2015, Kammon et al. (2018)
\end{abstract}


reported another ND outbreak in Alzintan city that emerged in backyard chickens and pigeons.

The results of Kammon et al. (2018) showed two viruses are genetically similar to those were isolated from cloacal swabs of backyard chickens in 2013, with a velogenic type of APMV-1 belongs to genotype VIb in a pigeon. Based on these results, it may indicate that NDV is still circulating in backyard birds which usually not vaccinated. Moreover, there is a lack of data about backyard poultry rearing and populations as well as their disease status which has resulted in a knowledge gap in understanding the epidemiological pattern of ND among backyard poultry. Therefore, the current study was conducted to measure the seroprevalence and molecular detection of NDV in backyard chickens in Tripoli, Libya.

\section{Materials and Methods}

\section{Samples collection and preparation}

A total number of 280 cloacal swabs were collected from non-vaccinated backyard chicken flocks in different geographical locations within the area of great Tripoli namely Qasr Ben Ghashier, Al-Sawani, Souq Al-Gomaa, Tajourah, Ein Zara, and Janzour (Table 1). Among those, 190 samples were collected during summer 2016 and 90 samples were collected at early winter 2016. A total of 412 blood samples were also collected from unvaccinated backyard chickens from the same geographical locations within the areas of great Tripoli. Swabs were stored on ice inside an ice box and serum separated from blood till transferred to the laboratory. The swabs were transferred under complete aseptic conditions into sterile McCartney (Bijou) glass tubes that contain standard transport medium then further stored at deep freezing $\left(-70^{\circ} \mathrm{C}\right)$ till being tested.

Data recording on web/smart phones (EpiCollect) EpiCollect (https://five.epicollect.net) is a mobile data-gathering platform for recording data pertaining to infectious diseases of certain animal populations including viral outbreaks affecting poultry populations. EpiCollect consists of two parts; a mobile application and a web server application. All records were obtained from chicken farms using this mobile phone application. Data included owner's name, number of samples, date of collection, type of vaccine, and place of collection. The locations included in current study were Qasr Ben Ghashier, Al-Sawani, Souq Al-Gomaa, Tajourah, Janzour and Ein Zara in Tripoli, Libya.

\section{Real time RT-PCR (rRT-PCR)}

RNA was extracted from all tracheal and cloacal samples by RNA Isolation Kit ME-0010/ME-0012 ZJ., Biotech and QIAamp Viral RNA Mini extraction Kit, QIAGEN as per the manufacturer's instructions. After extraction of RNA, samples were examined for APMV1 by rRT-PCR using a commercial kit (AnDiatec, Kornwestheim, Germany) and using the real-time protocol as previously described (Cattoli et al., 2009).

\section{Serological test of NDV using indirect ELISA}

Collected sera were tested for the presence of specific antibodies against NDV by ELISA commercial kit (CK116 NDV, BioChek, B.V, The Netherlands). The test was performed as per the manufacturer's instructions.

\section{Statistical analysis}

Prevalence of ND in different regions and seasons was compared using Chi-square test (SPSS Inc. Chicago, IL). The value of $(p<0.01)$ was taken as the cut-off value to consider differences statistically significant.

\section{Results}

\section{rRT-PCR results for NDV}

Data pertaining to the detection of NDV in backyard chickens and ducks during summer season (June) and early winter (November) as well as the locations are shown in Table 2 and Figure 1. The prevalence of NDV infection in backyard chickens in different locations of

Table 1. Number of samples (cloacal swabs) collected from backyard chickens in different locations of Tripoli.

\begin{tabular}{lccccccc}
\hline \multirow{2}{*}{ Season } & \multicolumn{5}{c}{ Locations } & \multicolumn{2}{c}{ Total } \\
\cline { 2 - 6 } & Qasr Ben Ghashier & Al-Sawani & Souq Al-Gomaa & Tajourah & Ein Zara & Janzour & \\
\hline Summer & 65 & 34 & 18 & 24 & 22 & 27 & 190 \\
Winter & 14 & 22 & 11 & 43 & 0 & 0 & 90 \\
Total & 79 & 56 & 29 & 67 & 22 & 27 & 280 \\
\hline
\end{tabular}

Table 2. Results of NDV for cloacal swabs of backyard chicken from Tripoli using rRT-PCR.

\begin{tabular}{|c|c|c|c|c|c|c|c|}
\hline \multirow{3}{*}{ Season } & \multicolumn{6}{|c|}{ Locations $^{\mathrm{a}}$} & \multirow{3}{*}{ Total Positive } \\
\hline & \multicolumn{2}{|c|}{ Qasr Ben Ghashier } & \multicolumn{2}{|c|}{ Al-Sawani } & \multicolumn{2}{|c|}{ Tajourah } & \\
\hline & Tested & +ve $(\%)$ & Tested & +ve $(\%)$ & Tested & +ve $(\%)$ & \\
\hline Summer & 65 & $60(92 \%)$ & 34 & $30(88 \%)$ & 24 & $14(58 \%)$ & $104 / 190(55 \%)$ \\
\hline Winter & 14 & $13(93 \%)$ & 22 & $5(23 \%)$ & 43 & $3(9 \%)$ & $21 / 90(23 \%)$ \\
\hline Total & 79 & $73(92 \%)$ & 65 & $35(54 \%)$ & 67 & $17(25 \%)$ & $125 / 280(45 \%)$ \\
\hline
\end{tabular}

${ }^{\text {a}}$ Samples collected from Janzour, Souq Al-Gomaa, and Ein Zara in summer and winter were negative for NDV by rRT-PCR. 
Tripoli during summer and winter was $45 \%$ using rRTPCR. In summer, 104 (55\%) out of 190 cloacal samples collected from chickens, were positive to NDV. The positive samples were distributed as $60(92 \%), 30$ (88\%), and 14 (58\%) from Qasr Ben Ghashier, AlSawani and Tajourah, respectively.

In the early winter, $21(23 \%)$ out of 90 samples collected from backyard chickens were found positive to NDV. The positive samples were distributed as $13(93 \%), 5$ $(23 \%)$, and $3(9 \%)$ from Qasr Ben Ghashier, Al-Sawani and Tajourah, respectively.

The prevalence in summer season was significantly higher than in winter $\left(\mathrm{X}^{2}=46.13, p \geq 0.00001\right)$. However, there were no significant differences in the prevalence of ND in Qasr Ben Ghashier in both seasons. Samples collected from Janzour, Souq Al-Gomaa, and Ein Zara in summer and winter were negative to NDV. Serological test results of NDV using indirect ELISA

Data pertaining to detection of antibodies against NDV by indirect ELISA are presented in Table 3 and Figure 2. ELISA test revealed 218 positive out of 412 tested samples with total prevalence of 53\% across the city of Tripoli in all regions. Obviously, Qasr Ben Ghashier has presented the highest prevalence of NDV specific antibodies (82\%) followed by Tajourah $(68 \%)$. The prevalence in Qasr Ben Ghashier was significantly higher as compared with other regions $\left(X^{2}=74.09, p \geq 0.00001\right)$. The prevalence of $49 \%$ and $41 \%$ were recorded in Ein Zara and Janzour, respectively.

\section{Prevalence (\%)}

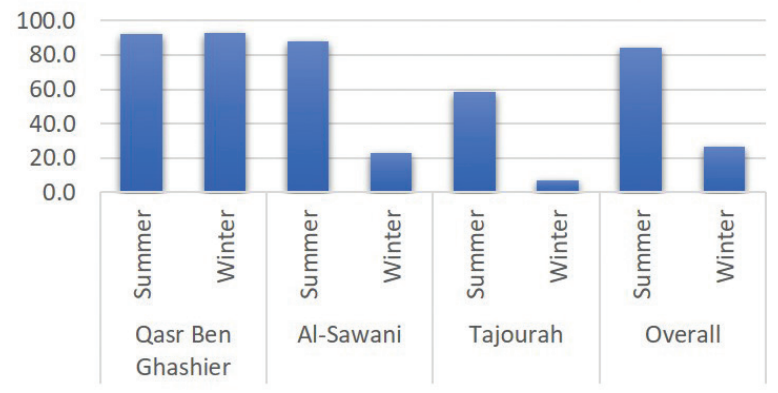

Fig. 1. The prevalence of NDV infection using rRT-PCR in backyard chickens in different locations of Tripoli during summer and winter.
The lowest prevalence was recorded in Souq Al-Gomaa (21\%).

\section{Discussion}

ND is a highly contagious viral disease affecting birds in many areas of the world and known as the most economically important disease that has a greater negative impact on poultry industry mainly due to high mortality (up to $80 \%-100 \%$ ) of infected chickens and decrease of egg production (Capua and Alexander, 2006).

The present study revealed a serious prevalence of ND in backyard chickens in different location of Tripoli by PCR and ELISA. The prevalence of NDV infection in backyard chickens in Qasr Ben Ghashier, Al-Sawani, Souq Al-Gomaa, Tajourah, Janzour and Ein Zara during summer and winter was $45 \%$ using rRT-PCR and 53\% using ELISA. ND recurrent outbreaks are common in Libyan poultry farms from time to time which indicates a possible endemic situation that have developed through a long term cumulative situation (Al-Garib et al., 2007). Few reports have documented that NDV may be contributed in an outbreak of a respiratory infection of multi-agents in broiler flocks in Tripoli, Libya (Al-Garib et al., 2006). Joseph et al. (2014) reported a prevalence rate of $17 \%$ in local chicken in the Federal Capital Territory, Abuja, Nigeria. ND is also still a major problem for poultry industry in Egypt. Deduced amino acid sequences and phylogenetic analysis of F gene of NDV revealed the circulation of NDV class II genotype VIId in Egypt during 2014 and 2015 outbreaks (Abd El Aziz et al., 2016). The level of NDV infection affecting the poultry farms in Algeria was $44 \%$ (Berghiche et al., 2018).

In the current study, the prevalence in summer $(55 \%)$ was higher than the prevalence in winter $(23 \%)$ by rRT-PCR. This result is in agreement with Munmun et al. (2016) who stated highest (50.6\%) prevalence of ND in summer season compared to lowest $(10.2 \%)$ in rainy season. It was previously reported that ND epidemics usually occur at times of climatic stress, leading to seasonal occurrence (Awan et al., 1994). Moreover, migratory birds are thought to play role in the transmission of avian viruses like NDV (Dhama et al., 2008). Some wild birds, specifically pigeons and

Table 3. Prevalence of NDV specific antibodies by ELISA among backyard chickens from different regions of Tripoli.

\begin{tabular}{lcccc}
\hline \multirow{2}{*}{ Regions } & $\begin{array}{c}\text { Total number of } \\
\text { samples }\end{array}$ & \multicolumn{3}{c}{ Positive samples } \\
\cline { 3 - 5 } & 84 & Number & \% from region & \% from total samples \\
\hline Tajourah & 82 & 57 & 68 & 26 \\
Janzour & 80 & 34 & 41 & 16 \\
Souq Al-Gomaa & 88 & 17 & 21 & 8 \\
Qasr Ben Ghashier & 78 & 72 & 82 & 32 \\
Ein Zara & 412 & 38 & 49 & 18 \\
Total & & 218 & $53^{\circ}$ & 100 \\
\hline
\end{tabular}




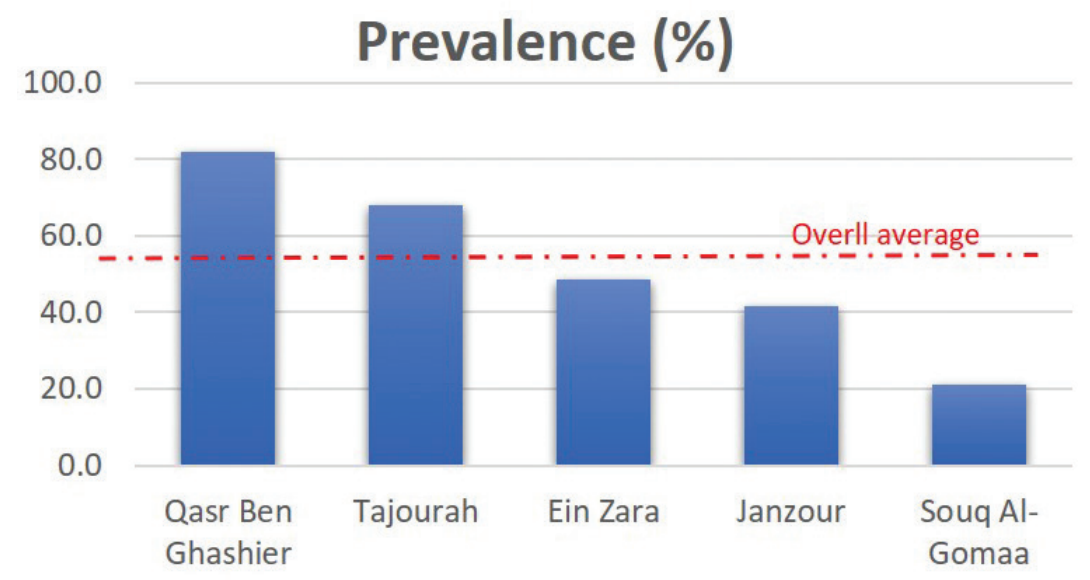

Fig. 2. The prevalence of NDV antibodies using ELISA in backyard chickens in different locations of Tripoli during summer and winter.

doves, could play a role as a reservoir hosts or carriers of virulent strains of NDV (Pedersen et al., 2016). Aquatic wild birds are known to migrate from Europe to Africa during months of August, September, and October every year and return back during summer months (Hahn et al., 2009; Hill et al., 2016). Epidemiological study was conducted in Farwa, Libya during period of 1998-2010, shows that a total of 41 species of migratory aquatic birds were recorded in Farwa island located at the West of Libya during the period from 1998 to 2010 (Essghaier et al., 2013). Backyard chickens in Libya usually kept in open areas where there are a great chance to be in contact with migratory and wild birds. It is notable that most infections with avian influenza and ND in Libya were reported in spring and early summer. In March 2013 and 2015, the Libyan poultry industry faced severe outbreaks caused by APMV-1 leading to high mortality and great economic losses in commercial and backyard birds (Kammon et al., 2015, 2018).

Among different locations, the prevalence of NDV infections was higher in Qasr Ben Ghashier (about 92\%) in summer and winter compared with other locations. The result of ELISA test for sera collected from Qasr Ben Ghashier was also the highest (82\%), which was statistically significant as compared with other regions $\left(X^{2}=74.09, p \geq 0.00001\right)$. In Tripoli, many locations are well known for their heavy density of commercial poultry farms as well as backyard poultry rearing. Most of commercial poultry farms in Tripoli are located in Qasr Ben-Ghashier, Tajourah and Al-sawany. More than thirty commercial and backyard poultry farms are found in a total area of $9 \mathrm{~km}^{2}$ in Qasr Ben-Ghashier. The uncontrolled rearing of backyard poultry has resulted in disastrous spread of viral diseases in many countries throughout the world (WHO/OIE/FAO, 2009). Samples collected from Janzour, Souq Al-Gomaa, and Ein Zara in summer and winter were negative for NDV by rRTPCR. However, sera collected from those locations and tested by ELISA were positive.
Out of 412 serum samples screened in this study by ELISA, 218 were positive representing 53\%. These results indicate that NDV specific antibodies were detected among backyard chickens. Backyard poultry are not vaccinated against ND, the presence of NDV antibodies in their sera would be as a result of natural infection with the virus. In addition, higher antibody titers of NDV in birds are likely due to natural infection which is known to be higher than antibodies titers that result from vaccination.

In spite of vigorous vaccination schedules, ND is still a threat to the poultry industry in developing countries (Luc et al., 1992). In Libya, vaccination could be as an effective method of controlling ND in commercial poultry, however, the veterinary services do not implement any vaccination program for backyard poultry.

Moreover, the use of poultry manure collected from commercial poultry farms as a natural fertilizer without previous heat treatment/suitable air drying can represent a potential source of the virus. Inter-state trade of poultry and poultry products is also a common feature, especially on known market days (Awan et al., 1994).

\section{Conclusion}

In conclusion, it is worthy to mention that the rare data about backyard poultry rearing as well as their diseases has resulted in a lack of awareness about the epidemiological pattern of ND among Libyan indoor poultry populations. This study demonstrated the situation of ND in backyard chicken as a source of infection highlighting the necessity of a comprehensive vaccination plan for backyard chickens.

\section{Acknowledgments}

The authors would like to thank the laboratory personnel of the National Center of Animal Health for the help and support. 


\section{Conflicts of interest}

The authors declare that they have no competing interests.

\section{Authors' contributions}

EAG, HAA, SOA and IME have designed the study. ASD did the statistical analysis. All authors have written, revised and approve the manuscript.

\section{References}

Abd El Aziz, M.N., Abd El-Hamid, H.S., Ellkany, H.F., Nasef, S.A., Nasr, S.M. and El-Bestawy, A.R. 2016. Biological and molecular characterization of newcastle disease virus circulating in chicken Flocks, Egypt, during 2014-2015. Zagazig Vet. J. 44(1), 9-20.

Alexander, D.J. 1997. Newcastle disease and other paramyxorius infections. In Diseases of poultry, 10th ed. Eds., Calnek, B.W., Barnes H.J., Beard C.W., Reid, W.M. and Jorder H.W. Ames, IA: Iowa State University Press, pp: 541-569.

Alexander, D.J. and Senne, D.A. 2008. Newcastle disease, other avian paramyxoviruses, and pneumovirus infections. In Diseases of poultry, 12th ed. Eds., Saif, Y.M., Fadly, A.M., Glisson, J.R., McDougald, L.R., Nolan, L.K. and Swayne, D.E. Ames, IA: Iowa State University Press, pp: 75-100.

Al-Garib, O.S., Kammon, A., Asheg, A., Hamid, M., Fathlla, H. and Lawal, A. 2007. Detection of antibodies to avian influenza H9N2 in broiler flocks clinically expressing a respiratory disease syndrome. In Proceeding of the 15th Congress $\&$ Exhibition of the World Veterinary Poultry Association. Beijing, China.

Al-Garib, S.O., Asheg, A.A., Kammon, A. and Hamid, M.A. 2006. An outbreak of a respiratory infection of multi-agents occurred in poultry flocks in Tripoli, Libya. In EPC 2006-12th European Poultry Conference, 2006 Sep 10-14, Verona, Italy, World's Poultry Science Association (WPSA).

Awan, M.A., Otte, M.J. and James, A.D. 1994. The epidemiology of Newcastle disease in rural poultry: A review. Avian Pathol. 23(3), 405-423.

Berghiche, A., Khenenou, T., Kouzi, A. and Labiad, I. 2018. An investigation on the predominant diseases, its diagnosis, and commonly used drugs in the poultry farms in the NorthEastern regions of Algeria. Vet. World 11(7), 986-989.

Capua, I. and Alexander, D.J. 2006. The challenge of avian influenza to the veterinary community. Avian Pathol. 35(3), 189-205.

Cattoli, G., De Battisti, C., Marciano, S., Ormelli, S., Monne, I., Terregino, C. and Capua, I. 2009. False-negative results of a validated real-time PCR protocol for diagnosis of Newcastle disease due to genetic variability of the matrix gene. J. Clin. Microbiol. 47(11), 3791-3792.
Dhama, K., Mahendran, M. and Tomar, S. 2008. Pathogens transmitted by migratory birds: Threat perceptions to poultry health and production. Int. J. Poult. Sci. 7(6), 516-525.

Doyle, T.M. 1927. A hitherto unrecorded disease of fowls due to a filter-passing virus. J. Comp. Pathol. Ther. 40(2), 144-169.

Essghaier, M.F.A., Etayeb, K.S., Bourass, E. and Prashant, J.J. 2013. Status and distribution of coastal birds at Farwa Island, Libya. Travaux de l'Institut Scientifique, Rabat, Série Zoologie 49(1), 79-85.

Hahn, S., Bauer, S. and Liechti, F. 2009. The natural link between Europe and Africa: 2.1 Billion Birds on Migration. OIKOS. 118(4), 624-626.

Hill, N.J., Ma, E.J., Meixell, B.W., Lindberg, M.S., Boyce, W.M. and Runstadler, J.A. 2016. Transmission of influenza reflects seasonality of wild birds across the annual cycle. Ecol. Lett. 19(8), 915-925.

Joseph, A.O., Sulaiman, L.K., Meseko, C.A., Ismail, S., Suleiman, I., Ahmed, S.J. and Onate, E.C. 2014. Prevalence of Newcastle disease antibodies in local chicken in federal capital territory, Abuja, Nigeria. Int Scholarly Res Notices, 2014, 3. https://doi. org/10.1155/2014/796148.

Kammon, A., Heidari, A., Dayhum, A., Eldaghayes, I., Sharif, M., Monne, I., Cattoli, G., Asheg, A., Farhat, M. and Kraim, E. 2015. Characterization of avian influenza and Newcastle disease viruses from Poultry in Libya. Avian Dis. 59(3), 422-430.

Kammon, A., Monne, I., Asheg, A. and Cattoli, G. 2018. Molecular detection and characterisation of avian paramyxovirus type 1 in backyard chickens and Pigeons in Alzintan City of Libya. Open Vet. J. 8(4), 401-405.

Kraneveld, F.C. 1926. A poultry disease in the Dutch East Indies. Nederlands-Indisch Bladen Voor Diergeneeskunde 38(1), 448-450.

Luc, P.V., Hong, N.T. and Chinh, V.T. 1992. Level of anti-Newcastle disease virus antibodies in industrial poultry at various ages and seasons. Agri. Food Ind. 9(1), 348-350.

Munmun, T., Islam, K.M.F., Jalal, S., Das, T., Tofazzol, R., Islam, K. Alam, R. 2016. Investigation of proportionate prevalence of Newcastle disease in chicken, pigeon and duck at selected veterinary hospitals in Bangladesh and India. J. Dairy Vet. Anim. Res. 4(2), 00118.

Pedersen, K., Marks, D.R., Afonso, C.L., Stopak, S.R., Williams-Coplin, D., Dimitrov, K.M., Miller, P.J. and DeLiberto, T.J. 2016. Identification of avian paramyxovirus serotype-1 in wild birds in the USA. J. Wildl. Dis. 52(3), 657-662.

Tiwari, A.K., Kataria, R.S., Nanthakumar, T., Dash, B.B. and Desai, G. 2004. Differential detection of Newcastle disease virus strains by degenerate primers based RT-PCR. Comp. Immunol. Microbiol. Infect. Dis. 27(3), 163-169. 
WHO/OIE/FAO. 2009. H5N1 Evolution Working Group. 2009. Letter to the Editor: Continuing progress towards a unified nomenclature for the highly pathogenic $\mathrm{H} 5 \mathrm{~N} 1$ avian influenza viruses: divergence of clade 2.2 viruses. Influenza Other Resp. Virus. 3(2):, 59-62. Available via http://www3.interscience.wiley.com/cgi-bin/ fulltext/122211969/HTMLSTART. 